CASE REPORT

\author{
K.J. Brown \\ V. Jewells \\ H. Herfarth \\ M. Castillo
}

\section{White Matter Lesions Suggestive of Amyotrophic Lateral Sclerosis Attributed to Celiac Disease}

\begin{abstract}
SUMMARY: $C D$ is an autoimmune-mediated disorder of the gastrointestinal tract. Initial symptom presentation is variable and can include neurologic manifestations that may comprise ataxia, neuropathy, dizziness, epilepsy, and cortical calcifications rather than gastrointestinal-hindering diagnosis and management. We present a case of a young man with progressive neurologic symptoms and brain MR imaging findings worrisome for ALS. During the diagnostic work-up, endomysium antibodies were discovered, and CD was confirmed by upper gastrointestinal endoscopy with duodenal biopsies. MR imaging findings suggestive of ALS improved after gluten-free diet institution.
\end{abstract}

ABBREVIATIONS: ALS = amyotrophic lateral sclerosis; $C D=$ celiac disease; FLAIR = fluid-attenuated inversion recovery; $\lg A=$ immunoglobulin $A$
C $\mathrm{D}$ is an inflammatory condition of the small intestine also called celiac sprue, nontropical sprue, and gluten-sensitive enteropathy. The most common presenting symptoms include diarrhea, steatorrhea, weight loss, iron deficiency anemia, and abdominal distension. Imaging findings specific to the brain have been reported primarily involving the periventricular white matter.

\section{Case Report}

A 32-year-old man with a 1-year history of balance difficulties presented with 1 week of worsening symptoms, including hand tremors and gait disturbance. Admission review of systems revealed a $60-$ pound $(27.22 \mathrm{~kg})$ weight loss during the past year, which he attributed to diarrhea consisting of 3-4 loose stools per day. Physical examination showed a broad-based gait leaning to the right, dysmetria with right finger-to-nose, hyperreflexia, an upgoing right toe, right lower extremity weakness, and right foot drop. Sensation was normal. Laboratory studies revealed a slightly elevated phosphorus concentration $(4.8 \mathrm{mg} / \mathrm{dL})$, but findings were otherwise normal. Brain MR imaging showed abnormal increased signal intensity on T2 and FLAIR in the bilateral corona radiata, extending inferiorly into the corticospinal tracts without contrast enhancement (Fig 1).

Initial diagnostic considerations included ALS and Friedrich ataxia. Electromyelography findings of the right upper and lower extremities were normal. Total spine MR imaging and CT findings of the chest, abdomen, and pelvis were normal. CSF findings for herpes simplex virus, human herpesvirus 6 cytomegalovirus, varicella-zoster virus, Epstein-Barr virus, and Venereal Disease Research Laboratory test were negative, but +4 oligoclonal bands were noted. Findings were negative for paraneoplastic antibodies. Ataxia work-up included a positive antiendomysial antibody titer of 1:160. Duodenal biopsies yielded crypt hyperplastic villous atrophy with numerous intraepithelial lymphocytes, most consistent with gluten-sensitive enteropathy.

The patient started a strict gluten-free diet with improvement of symptoms during the next several months. Repeat brain MR imaging (23 months after initial presentation) revealed resolution of initial

Received June 23, 2009; accepted July 3.

From the Department of Radiology (K.J.B., V.J., M.C.) and Division of Gastroenterology and Hepatology (H.H.), University of North Carolina, Chapel Hill, North Carolina.

Please address correspondence to Kelan J. Brown, MD, Department of Radiology, University of North Carolina, 2036 Old Clinic Bldg, CB 7510, Chapel Hill, NC, 27599-7510; e-mail: kelanbrown@gmail.com

DOI 10.3174/ajnr.A1826 abnormalities (Fig 2). Continued physical therapy and a gluten-free diet have been prescribed.

\section{Discussion}

$\mathrm{CD}$ is an inflammatory condition of the small intestine also called celiac sprue, nontropical sprue, and gluten-sensitive enteropathy. ${ }^{1}$ The disease appears in early childhood and has a second peak in the third-to-fourth decades. Males and females are equally affected, and its incidence has increased in Northern Europe and the United States. ${ }^{2,3}$ Multiple other disorders have been associated with $\mathrm{CD}$, including Down syndrome, insulin-dependent diabetes mellitus, IgA deficiency, and rheumatoid arthritis. ${ }^{1}$

Symptoms are precipitated in genetically predisposed individuals by ingestion of foods containing gluten, such as wheat, rye, and barley. Consumption of triggering foods leads to an autoimmune-mediated inflammatory response in the small intestine, resulting in villous atrophy and malabsorption. ${ }^{3}$ The most common presenting symptoms include diarrhea, steatorrhea, weight loss, iron deficiency anemia, and abdominal distension.

The diagnosis is sometimes difficult to make given that not all patients present in the same manner and that symptoms overlap other gastrointestinal disorders. Half of patients have iron deficiency anemia or osteoporosis, without diarrhea. Diagnostic evaluation for CD consists of antibody testing and duodenal biopsy. ${ }^{4}$ The most sensitive and specific antibodies for its confirmation are tissue transglutaminase IgA and endomysial IgA antibodies. Due to the low specificity compared with tissue transglutaminase and endomysium antibodies, gliadin antibodies should no longer be used in the diagnostic work-up for CD. Duodenal biopsies are still the criterion standard for tissue diagnosis and reveal villous atrophy ${ }^{5}$ and increased intraepithelial lymphocytes. ${ }^{6}$

Neurologic findings associated with CD disease were initially reported in 1966 by Cooke and Smith. ${ }^{7}$ Most common symptoms are ataxia and neuropathy, followed by epilepsy, ALS and multiple sclerosis-like symptoms, isolated motor neuron disorders, sensory ataxia, and dizziness. These complications may arise as consequences of vitamin and mineral deficiencies. Other authors discuss a potential pathophysiologic role of antigliadin antibodies in a neurotoxic autoimmune process. $^{8}$ 

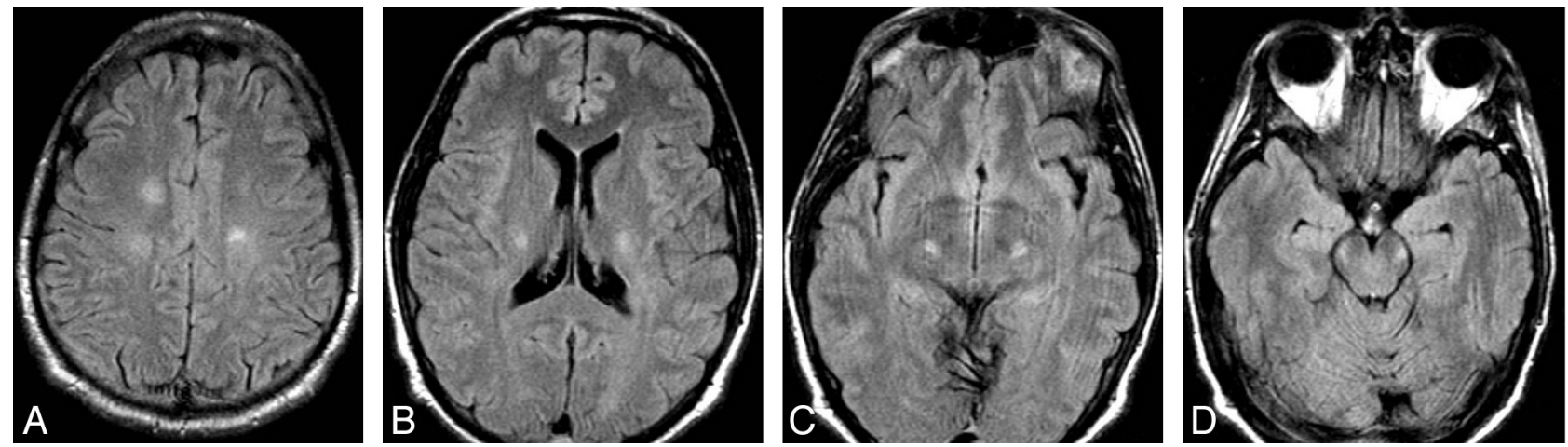

Fig 1. FLAIR images from initial brain MR imaging demonstrate abnormal increased signal intensity in the white matter of the centra semiovale and bilateral corticospinal tracts.
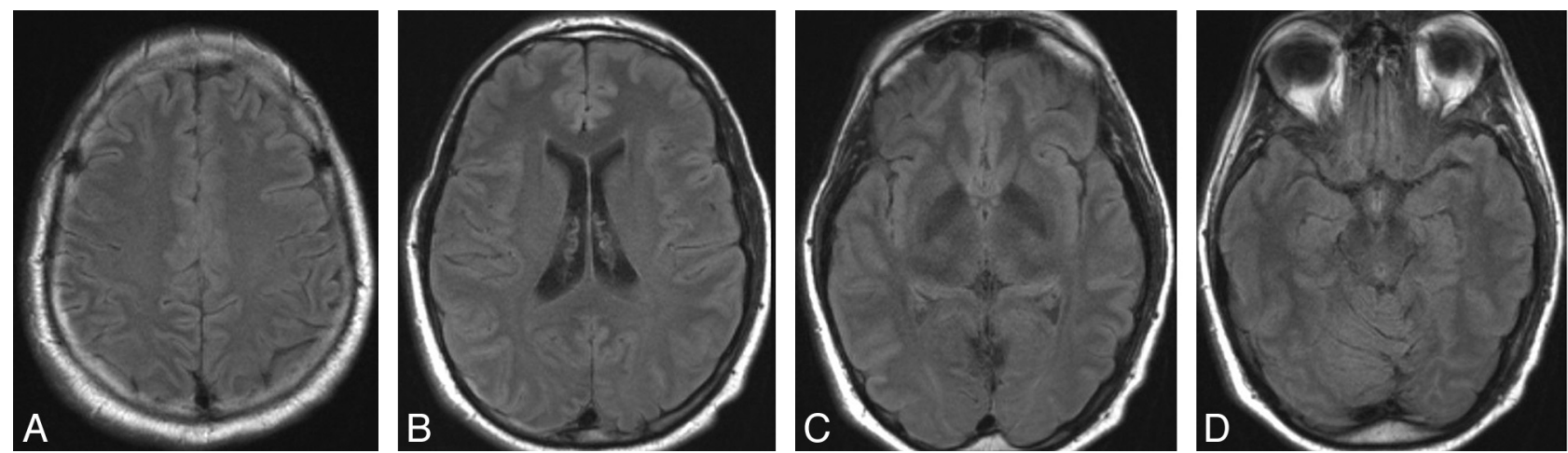

Fig 2. Follow-up FLAIR images after a gluten-free diet show complete resolution of signal-intensity abnormalities.

MR imaging is used primarily in the diagnostic work-up of $\mathrm{CD}$-associated conditions. Nonspecific periventricular white matter lesions of various sizes with increased T2/FLAIR signal intensity and without enhancement have been described. ${ }^{9,10}$ The lesions may be unilateral and bilateral. ${ }^{11}$ Occipital calcifications have also been reported. In 2007, Turner et al ${ }^{12}$ reported a case of CD mimicking ALS with T2/FLAIR signalintensity abnormalities in the left corticospinal tract, which improved on a gluten-free diet. Our patient also showed abnormal signal intensity involving both corticospinal tracts on MR imaging. His symptoms and MR imaging lesions improved and resolved, respectively, on appropriate treatment of $\mathrm{CD}$. Ours is the second patient reported with this group of findings. Because ALS is a progressive and untreatable disease while $\mathrm{CD}$ is easily treatable, considering the latter as a cause of neurologic disorders in patients with ALS-like symptoms may be indicated.

\section{References}

1. Green PH, Cellier C. Celiac disease. N Engl J Med 2007;357:1731-43

2. Collin P, Kaukinen K, Vogelsang H, et al. Antiendomysial and antihuman recombinant tissue transglutaminase antibodies in the diagnosis of coeliac disease: a biopsy-proven European multicentre study. Eur J Gastroenterol Hepatol Jan 2005; 17:85-91

3. Hadjivassiliou M, Williamson CA, Woodroofe N, et al. The immunology of gluten sensitivity: beyond the gut. Trends Immunol 2004;25:278-82

4. Mandal A, Mehdi I, Munshi S, et al. Value of routine duodenal biopsy in diagnosing coeliac disease in patients with iron deficiency anaemia. Postgrad Med J 2004;80:475-77

5. Brocchi E, Corazza GR, Caletti G, et al. Endoscopic demonstration of loss of duodenal folds in the diagnosis of celiac disease. N Engl J Med 1988;319: 741-44

6. Sollid L. Intraepithelial lymphocytes in celiac disease: license to kill revealed. Immunity 2004;21:303-04

7. Cooke WT, Smith WT. Neurological disorders associated with adult celiac disease. Brain 1966;89:683-722

8. Hadjivassiliou, M, Gibson A, Davies-Jones CA, et al. Does cryptic gluten sensitivity play a part in neurological illness? Lancet 1996;347:369-71

9. Hadjivassiliou M, Grünewald RA, Lawden M, et al. Headache and CNS white matter abnormalities associated with gluten sensitivity. Neurology 2001; $56: 385-88$

10. Kieslich M, Errázuriz G, Posselt HG, et al. Brain white-matter lesions in celiac disease: a prospective study of 75 diet-treated patients. Pediatrics 2001;108:E21

11. Lea ME, Harbord M, Sage MR. Bilateral occipital calcification associated with celiac disease, folate deficiency, and epilepsy. AJNR Am J of Neuroradiology 1995; 16:1498-500

12. Turner M, Chocan G, Quaghebeur G, et al. A case of celiac disease mimicking amyotrophic lateral sclerosis. Nat Clin Pract Neurol 2007;3:581-84 